\title{
EchoGéo
}

39 | 2017

Ressources urbaines (1)

\section{Identifier les ressources urbaines pour lire les inégalités socio-spatiales}

Introduction

\section{Aurélia Michel and Antonine Ribardière}

\section{OpenEdition}

\section{Journals}

Electronic version

URL: https://journals.openedition.org/echogeo/14925

DOI: $10.4000 /$ echogeo. 14925

ISSN: 1963-1197

Publisher

Pôle de recherche pour l'organisation et la diffusion de l'information géographique (CNRS UMR 8586)

Electronic reference

Aurélia Michel and Antonine Ribardière, "Identifier les ressources urbaines pour lire les inégalités socio-spatiales", EchoGéo [Online], 39 | 2017, Online since 28 March 2017, connection on 10 August 2021. URL: http://journals.openedition.org/echogeo/14925 ; DOI: https://doi.org/10.4000/echogeo. 14925

This text was automatically generated on 10 August 2021

EchoGéo est mis à disposition selon les termes de la licence Creative Commons Attribution - Pas d'Utilisation Commerciale - Pas de Modification 4.0 International (CC BY-NC-ND) 


\title{
Identifier les ressources urbaines pour lire les inégalités socio- spatiales
}

\author{
Introduction
}

\author{
Aurélia Michel and Antonine Ribardière
}

1 Depuis plusieurs années, des travaux permettent de renouveler la qualification des processus sociaux qui accompagnent la forte urbanisation des sociétés au Nord comme au Sud, notamment la production d'inégalités. Ceux-ci se fondent sur la qualification et la caractérisation des ressources qui sont en jeu dans ces processus: logement et prestations attenantes, emploi, école, santé, transports intra-urbains, mobilité ${ }^{1}$ ou au contraire immobilité2.

2 Nous proposons d'aborder ces ressources comme des ressources urbaines, c'est-à-dire non seulement comme une somme de services mais également selon leur répartition spécifique dans l'espace de la ville, leur articulation et leurs conditions d'accès. Cette approche permet de faire d'une localisation dans la ville une ressource en tant que telle, de même que l'accès à des lieux de ressources potentielles. En outre, elle permet d'introduire une dimension diachronique dans la qualification des ressources envisagées par leur localisation : les ressources urbaines sont en effet le résultat d'une construction, dans le temps et par des acteurs, publics et privés; elles sont l'objet de modifications importantes dans l'histoire de l'espace urbain (croissance de la ville, changements économiques, construction de nouvelles infrastructures, etc.).

3 S'il n'est pas simple de qualifier les ressources urbaines par des caractères objectifs et quantifiables, leur réalité empirique est mise en évidence dans des situations connues par ailleurs : par exemple pour constater leur insuffisance dans certains quartiers, ou encore à travers le marché immobilier, qui d'une certaine manière, quantifie ces ressources sans toutefois les définir. Les ressources urbaines, au-delà de la somme brute des prestations de service auxquelles accèdent les habitants, sont en particulier mobilisées pour qualifier des situations de marginalité ou de pauvreté, tant à partir des trajectoires individuelles que des espaces produits. 
4 Les ressources urbaines apparaissent ainsi comme des marqueurs des inégalités sociospatiales. La carence de services dans certains quartiers populaires ou au contraire, la densité de services rares dans des quartiers plus centraux, à l'origine de rentes de situations ${ }^{3}$ peuvent être lues à la fois comme un effet et comme un élément de la production des inégalités. Les filières d'accès aux logements, la structure du parc et le fonctionnement $\mathrm{du}$ marché foncier orientent et contraignent les stratégies résidentielles des ménages. Sur le front de l'emploi, les distances entre lieux de travail et lieux de résidence, les niveaux de desserte et la qualité des réseaux et enfin les coûts de transports, constituent une réelle entrave à l'accès à l'emploi des catégories populaires (Bertrand 2010, op. cit., WenglenskIi S., 2010).

5 La problématique de l'accès aux ressources urbaines se décline différemment selon les individus, non seulement suivant leur localisation propre - renvoyant en cela à des effets de contexte qui associent ressources spécifiques au quartier d'une part et situation du quartier dans la ville d'autre part ${ }^{4}-$, mais également selon les autres ressources que chacun est en mesure de mobiliser - ressources économiques, capacités à se déplacer, réseau social (Lévy J.-P., Brun J., 2000)...

6 Enfin, la notion de ressources exprime des moyens effectivement disponibles, mais elle peut aussi être comprise de manière relative : les ressources urbaines désignent alors une offre, potentiellement accessible. De fait, les enjeux d'accès à ces ressources et leur pérennisation font l'objet de stratégies à plusieurs échelles (familiales, communautaires, individuelles) qui sont reflétées par les trajectoires résidentielles et professionnelles des habitants. L'accès, ou au contraire l'impossibilité d'accéder à ces ressources traduit de manière concrète les conditions d'exercice d'une citoyenneté habitante dont la revendication traverse tant de mouvements sociaux et politiques publiques récentes. Comment donc les objectiver, afin de les prendre en compte dans la compréhension de la production des inégalités et des dynamiques sociales urbaines plus générales?

7 Ces questions sont d'autant plus cruciales lorsqu'elles s'appliquent à des contextes urbains dans lesquels les populations vulnérables représentent une part importante, voire majoritaire, des citadins. Il n'est donc pas anodin que les contributions reçues pour ce dossier portent toutes sur des terrains au Sud, où la question de l'accessibilité aux biens et aux services se pose de manière aiguë.

8 Au-delà de ce point commun, les contributeurs de ce numéro ont emprunté différentes entrées pour caractériser les ressources urbaines et les politiques qui peuvent y être associées : par la qualification des espaces urbains numéro 39); par les pratiques des acteurs, contraintes par leur statut ou leur lieu de résidence (numéro 39); par les systèmes de mobilités, qui déterminent l'accessibilité des ressources dans la ville (numéro 40); enfin par l'articulation entre les trajectoires des acteurs et les ressources du lieu (numéro 40).

\section{Répartition des ressources dans la ville et qualification des espaces urbains en cours de maturation}

9 Un premier ensemble de textes pose la question de la répartition des ressources dans la ville, en termes de densité, de carence ou encore d'accessibilité, afin de qualifier les 
espaces urbains en fonction non seulement de la présence ou concentration de ressources, mais aussi de la manière dont ces espaces s'articulent au reste de la ville. Ces articles abordent en particulier des quartiers intermédiaires et périphériques. Autrement dit, la trajectoire de ces quartiers est observée du point de vue de leurs ressources propres, mais aussi à travers la manière dont leurs habitants accèdent à ces ressources et à celle de l'ensemble de la ville.

Ainsi, avec une approche essentiellement quantitative, Eugênia Viana Cerqueira croise le potentiel des lieux, appréhendé à travers les équipements urbains, et celui des individus, saisi à partir des ressources économiques. L'auteure montre comment les deux se cumulent : les différentiels de potentiel entre les lieux sont encore renforcés par ceux entre les individus, d'autant plus que les inégalités mises en évidence recoupent des usages différenciés des modes de transports. Les périphéries urbaines qui se sont développées à Belo Horizonte (Brésil) se différencient ainsi non seulement suivant la composition socio-économique des habitants, mais également suivant les modes d'accès aux ressources urbaines et les pratiques de mobilités. L'article met en évidence une dépendance des plus pauvres vis-à-vis du centre et de manière générale, une mobilité subie pour accéder aux ressources. La diversification sociale des franges périphériques reproduit, en le complexifiant mais sans l'atténuer, le modèle d'urbanisation ségrégatif.

11 Une autre approche pour caractériser la situation des quartiers périphériques populaires en termes d'accès aux ressources urbaines est proposée par Jean-François Valette à propos des colonias populares de Mexico (Mexique). En partant dans un premier temps des pratiques de mobilité des habitants de ces périphéries, l'auteur révèle les ressources urbaines qui existent autour de la résidence, liées notamment à la densité des activités économiques informelles et également à l'ancrage des réseaux de sociabilité, qui renvoie alors à la dimension foncière du peuplement, tout autant qu'à celle de l'organisation sociale. L'analyse des pratiques permet en outre de qualifier la manière dont les habitants accèdent aux ressources du centre ou à celles des quartiers voisins. Il apparait que les colonias populares sont, plus qu'on ne pourrait le penser, articulées au reste de la métropole, les effets de relégation étant ainsi à nuancer. Enfin, il met en évidence la variété des pratiques à l'échelle du quartier : un même contexte résidentiel peut s'intégrer dans des systèmes de lieux de portée très variée, centrés sur le local ou au contraire développés à l'échelle métropolitaine. L'auteur souligne à cette occasion combien la pratique de la ville et le développement de compétences urbaines constituent des marqueurs de différenciation sociale importants, entre dépendance voire captivité relativement au contexte local, et ancrage citadin multipolarisé.

La diversité des espaces périphériques est également au cœur de la lecture des rapports de genre que proposent Vincent Berdoulay et Thi Thu Trang Ngo à Hô Chi Minh Ville (Vietnam). À travers une approche dialectique entre les ressources que les femmes sont en mesure de mobiliser pour voir évoluer les rapports de genre, et les ressources qui leur restent inaccessibles du fait des rapports de genre, les auteurs montrent comment l'accès à l'emploi constitue un vecteur puissant d'évolution d'une part, et également d'inégalité entre les femmes par le biais des statuts de l'emploi (salarié ou informel). Via les différences de rapports sociaux relevées entre types d'habitats, nous voyons ainsi apparaitre un espace de transition qui correspond à des transitions sociologiques majeures au Vietnam, dans lesquelles le rapport de genre peut aussi se redéfinir à 
travers les ressources urbaines: statut du logement, articulation ou conflit entre fonctions domestiques et emploi dans le contexte périurbain.

\section{Les ressources urbaines définies par les pratiques informelles}

Une autre manière de définir les ressources urbaines s'appuie sur l'analyse des pratiques de la ville par des acteurs qui sont démunis, partiellement ou complètement, des statuts et protections de la résidence voire de la citoyenneté. C'est le cas des migrants boliviens et paraguayens à São Paulo, dont traite l'article de Sylvain Souchaud, qui s'insèrent dans le secteur de la confection, une niche économique qui joue à la fois sur l'informalité et la localisation dans le centre de la métropole. Les trajectoires des migrants d'origine bolivienne et paraguayenne sont étroitement articulées avec l'organisation de ce secteur d'activité, qui leur fournit l'emploi et le logement dans la métropole. Toutefois cette médiation par les réseaux migratoires laisse peu de place à une insertion plus large dans la société urbaine d'accueil. L'auteur souligne ainsi le caractère paradoxal de l'expérience urbaine des primo-arrivants, entre exclusion du marché de l'emploi et du logement du fait de leur statut d'étranger et réalisation du projet migratoire à travers cette ressource propre à la migration que constitue l'atelier de confection.

Une même ambivalence traverse l'article de Laurent Faret, qui interroge l'accès à l'environnement urbain de migrants centraméricains à Mexico, dans des conditions extrêmes où les migrants d'installation récente, souvent à la faveur d'un projet migratoire contrarié, se retrouvent seuls face à la métropole et à ses ressources. Celles auxquels ils parviennent à accéder sont, dans un premier temps au moins, spécifiques à leur trajectoire migratoire. Ils s'adressent ainsi à des associations qui peuvent les aider dans leur transit plus ou moins prolongé et s'appuient également sur les caractéristiques mêmes de la métropole. En effet, l'articulation des réseaux de mobilité à différentes échelles (locales, régionales, nationales et internationales) sert leur stratégie de déplacement qui vise à éviter les contrôles, tout en accédant le plus directement possible aux lieux de passage de la frontière nord. Par la précarité de leur présence en ville, l'apprentissage des ressources du lieu reste très relatif, souvent limité à une échelle locale et fortement contraint par la vulnérabilité du statut migratoire.

L'informalité des favelas de Rio de Janeiro (Brésil), dans la perspective de Rafael Gonçalves, peut aussi constituer une ressource urbaine. En permettant la consolidation d'un habitat relativement proche des centres économiques, l'informalité des favelas produit un type spécifique de tissu urbain où la polyfonctionalité des bâtiments et la souplesse des formes d'occupation résolvent de nombreux paradoxes liés à la métropole et que les politiques publiques n'ont pas toujours, ou mal, pris en charge. Sans en nier l'ambiguïté, l'auteur montre l'importance des ressources de l'informalité et la complexité sociale qui lui est associée, ce que les opérations qui visent à formaliser ces pratiques doivent absolument prendre en compte.

Ces trois textes illustrent comment des individus au statut peu consolidé (en tant que citadin ou citoyen) n'ont d'une certaine manière à leur disposition que les ressources de la ville elle-même (centralité, diversité fonctionnelle, nœud et interface dans la circulation). De ce fait, ils pointent l'ambivalence de situations qui produisent 
également des formes de "captivité » pour ces populations, dans des niches délimitées par l'absence de statut, où ils restent exposés et vulnérables.

\section{Ressources urbaines, mobilité et accessibilité}

17 La question de la répartition des ressources dans la ville conduit à identifier les ressources nécessaires à leur accessibilité. Plusieurs articles de ce numéro s'y consacrent en abordant les systèmes de mobilité, confrontant politiques publiques et pratiques des habitants.

18 Les trois villes abordées de ce point de vue - Le Cap (Afrique du Sud), Bouaké (Côte d'Ivoire) et Santiago (Chili)-, en dépit de contextes démographiques, économiques et historiques différents, se caractérisent toutes les trois par une très forte pression sur les ressources $\mathrm{du}$ centre et par une carence ou un dysfonctionnement des infrastructures de transport qui permettraient une meilleure accessibilité pour le plus grand nombre d'habitants. Ainsi, Jean-Michel Kouakou Attien expose les difficultés concrètes de déplacement dans la deuxième ville de Côte d'Ivoire, Bouaké. La dégradation de l'offre existante après la crise politique de 2002 a été compensée par une nouvelle offre, plus souple mais aussi plus dangereuse pour les usagers : les mototaxis. Si cette offre se révèle indispensable pour dépasser le frein de la distance dans cette ville de plus de 500000 habitants, elle n'en constitue pas moins un vecteur de vulnérabilité pour les citadins - on touche ici de nouveau l'ambivalence des ressources auxquelles les plus démunis sont en mesure d'accéder.

La dichotomie centre/périphérie marque une autre pratique, dans un tout autre contexte : l'usage du vélo à Santiago du Chili. Claudia Arellano Yévenes et Fernando Saavedra Peláez montrent comment un même moyen de transport peut exprimer des ressources très différentes, qui renvoient à la localisation résidentielle des habitants. D'un côté le vélo recouvre une pratique alternative et choisie, associée à des déplacements courts pour les habitants du centre; de l'autre au contraire son usage témoigne d'une pratique contrainte et de l'absence d'alternative, associées à des déplacements longs, pour les habitants des périphéries. L'équipement des itinéraires centraux en voie propre illustre la manière dont les politiques publiques peuvent contribuer à accentuer les inégalités d'accès à la ville.

L'héritage du régime de l'apartheid en Afrique du Sud pèse encore lourdement sur le système de transport au Cap, analysé par Solène Baffi. En montrant comment historiquement, les réseaux et les modalités du transport ont reflété les objectifs de l'ancien régime ségrégationiste sud-africain, elle pose la question de la réorientation des politiques publiques de transport depuis la fin de l'apartheid et de leur capacité à constituer un vecteur d'inclusion socio-spatiale des populations. L'auteure montre que si le modèle néolibéral de gestion des transports publics, qui prévaut au Cap comme dans bien d'autres villes, a clairement amélioré la mobilité potentielle des populations dépendantes des transports en communs, l'accessibilité réelle de ces populations reste à construire, via le développement en particulier d'un système intermodal et intégré. 


\section{Les ressources urbaines entre aménités et pratiques}

21 L'accès aux ressources urbaines ne dépend pas que de leur localisation dans la ville, ni des seules conditions matérielles pour les atteindre. Entrent en jeu d'autres ressources, que les individus sont - ou non - en mesure de mobiliser. L'objet des deux derniers articles est de mettre en évidence l'agencement des ressources urbaines, de différents niveaux, dans les modalités d'insertion des migrants nationaux d'origine rurale dans la zone métropolitaine de Mexico. Antonine Ribardière montre que si cette migration s'appuie le plus souvent sur des relais, parents qui ont précédé le migrant dans sa trajectoire, les trajectoires résidentielles et professionnelles ne s'inscrivent toutefois pas dans le cadre de filières, ni en marge de la société urbaine dominante. La configuration particulière de Naucalpan, périphérie industrielle et résidentielle de Mexico qui attire la migration depuis des décennies, permet aux derniers arrivés d'articuler les enjeux de l'emploi et du logement, tandis que les migrants plus anciennement implantés s'affranchissent progressivement du cadre local et accèdent, plus largement, aux ressources de la métropole. Aurélia Michel montre ensuite comment les ressources urbaines dont ces populations sont dépourvues au départ deviennent rapidement un enjeu de leur trajectoire. Les récits de vie qui sont collectés dans l'article témoignent que ces ressources sont acquises petit à petit, au gré des arbitrages et projets familiaux qui privilégient tantôt l'accès à la centralité, tantôt l'ancrage et les ressources de la proximité.

Pour finir cette présentation, nous souhaitons pointer trois pistes de réflexion qui ressortent des conclusions des articles, et qui peuvent contribuer à préciser la définition des ressources urbaines.

Tout d'abord, celles-ci désignent avant tout à un agencement spatial de ressources. Dans cette acceptation restreinte, les ressources urbaines renvoient aux ressources de la ville elle-même, dont on a vu qu'elles étaient d'autant plus stratégiques qu'elles sont les seules à disposition d'individus dont le statut est vulnérable, sur un plan légal, économique ou social, et qui n'accèdent généralement pas aux ressources publiques. Deuxième point, le système de mobilité, et donc l'action publique, détermine en grande partie la relation entre l'agencement des ressources dans la ville et leur accessibilité permise par les capacités économiques, sociales et politiques des individus. Ces dernières ont aussi, troisième point, une dimension proprement urbaine lorsqu'elles permettent la maitrise de l'environnement urbain et donc de l'agencement spatial des ressources dans la ville. La notion de ressources urbaines permet ainsi de situer la réflexion sur les inégalités à l'intersection de deux logiques, celle qui détermine l'agencement spatial des ressources en ville et celle qui détermine la manière dont les citadins peuvent s'en saisir. Une réflexion sur l'apport de cette notion, en conclusion du dossier, est apportée par Monique Bertrand, à l'aune des discussions soulevées par les différents articles. 


\section{BIBLIOGRAPHY}

Authier J.- Y. (dir.) et al., 2001. Du domicile à la ville. Vivre en quartier ancien. Anthropos, coll. Villes, $214 \mathrm{p}$.

Bertrand M., 2010. Introduction. Revue Tiers Monde, $1 / 2010$ (n² 201), p. 7-23.

Bourdieu P., 1993. La Misère du monde. Ed. du Seuil, 956 p.

Fol S., 2010. Mobilité et ancrage dans les quartiers pauvres : les ressources de la proximité. Regards Sociologiques, $n^{\circ} 40$, p. 27-43.

Lévy J.-P., Brun J., 2000. De l'extension au renouvellement métropolitain : mosaïque sociale et mobilité. In Dureau F., Dupont V., Lelièvre E., Lévy J.P., Lulle T. (éd.), 2000. Métropoles en mouvement : une comparaison internationale. Paris, Anthropos-IRD, coll. Villes, p. 229-246.

WenglenskIi S., 2010. Accès à l'emploi : champ des possibles contre champ des pratiques. In Massot M.H. (dir.). Mobilités et modes de vie métropolitains. Paris, L'Oeil d'or, p. 121-137.

\section{NOTES}

1. Monique Bertrand relève ainsi : "Autrefois comprise comme un simple vecteur vers des ressources territorialisées, et liée à l'aménagement du territoire, la mobilité est désormais perçue elle-même comme ressource dans et de la ville » (Bertrand, 2010).

2. Sylvie Fol souligne ainsi l'importance des ressources de l'espace local pour les catégories populaires ((Fol, 2010).

3. Bourdieu décrit ces « profits de localisation » dans le chapitre « Effets de lieux » de La Misère du monde (Bourdieu, 1993).

4. Effets de contexte mis en évidence par exemple par Authier J.- Y. (dir.) et al. (2001).

INDEX

Subjects: Sur le Champ - Sur le Terrain

\section{AUTHORS}

\section{AURÉLIA MICHEL}

Aurélia Michel, aurelia.michel@univ-paris-diderot.fr, historienne est Maitre de conférences à

l'Université Paris Diderot.

\section{ANTONINE RIBARDIÈRE}

Antonine Ribardière, antonine.ribardiere@univ-paris1.fr, est Maître de conférences à

l'Université Paris 1 Panthéon-Sorbonne et membre de l'UMR 8586 PRODIG 